\title{
Complex Evolution of Coronal Mass Ejections in the Inner Heliosphere as Revealed by Numerical Simulations and STEREO Observations: A Review
}

\author{
Noé Lugaz ${ }^{1}$, Charles J. Farrugia ${ }^{1}$ and Nada Al-Haddad ${ }^{2}$ \\ ${ }^{1}$ Space Science Center and Department of Physics, University of New Hampshire, \\ Morse Hall, 8 College Rd, Durham, NH, 03824, USA \\ email: noe.lugaz@unh.edu \\ ${ }^{2}$ Center for Plasma Astrophysics, KU Leuven, Leuven, BE
}

\begin{abstract}
The transit of coronal mass ejections (CMEs) from the Sun to 1 AU lasts on average one to five days. As they propagate, CMEs interact with the solar wind and preceding eruptions, which modify their properties. In the past ten years, the evolution of CMEs in the inner heliosphere has been investigated with the help of numerical simulations, through the analysis of remote-sensing heliospheric observations, especially with the SECCHI suite onboard STEREO, and through the analysis of multi-spacecraft in situ measurements. Most studies have focused on understanding the characteristics of the magnetic flux rope thought to form the core of the CME. Here, we first review recent work related to CME propagation in the heliosphere, which point towards the need to develop more complex models to analyze CME observations. In the second part of this article, we review some recent studies of CME-CME interaction, which also illustrate the complexity of phenomena occurring in the inner heliosphere.
\end{abstract}

Keywords. Sun: coronal mass ejections (CMEs), solar-terrestrial relations, methods: numerical, methods: data analysis

\section{Introduction}

The heliospheric propagation of coronal mass ejections (CMEs) has historically been one of the "poor children" of space physics research as it appears to lack the fundamental physical questions at the core of the research about CME initiation, coronal heating, solar wind acceleration or geomagnetic storms and substorms. Early work in the 1970s and 1980s took advantage of the presence of multiple spacecraft measuring the solar wind at different heliocentric distances in the inner heliosphere. However, after the mid-1980s and the end of the Helios missions, most in situ measurements were made from $1 \mathrm{AU}$ or beyond. In the meantime, coronagraphic observations by SMM and SOHO have been providing remote-sensing views of CMEs up to distances of at most $0.15 \mathrm{AU}$, leaving the large majority of the inner heliosphere without direct remote-sensing observations or in situ measurements. In the past ten years, CMEs have been remotely imaged in the inner heliosphere by SMEI and the Heliospheric Imagers (HIs) onboard STEREO (Davies et al. 2009; Howard et al. 2013). In addition, multiple, simultaneous in situ measurements of CMEs at the same heliocentric distance but at different longitudes have been made possible by the presence of the two STEREO spacecraft and ACE and Wind at the L1 point. Other observations (interplanetary scintillation -IPS-, type II and type III radio bursts, etc.) can also be useful to obtain additional information about CMEs and CME-driven shocks. 
Most analyses of CMEs in the heliosphere rely on the concept of twisted magnetic flux ropes (TMFRs) to understand and describe their properties. In this way, one often refers to the CME width or radius, the orientation of its axis, etc. In fact, TMFR models of CMEs have been extremely successful to study CME properties in the corona (e.g., Chen 1996; Thernisien et al. 2006), and in the heliosphere (e.g., Lepping et al. 1990; Wood et al. 2009). They are also the foundation of many numerical and theoretical studies, where the TMFR is either initiated out of equilibrium (Manchester et al. 2004b), emerged from below the photosphere (Archontis et al. 2004), perturbed (Titov \& Démoulin 1999; Amari et al. 1999) or formed and destabilized (Linker \& Mikic 1995; Antiochos et al. 1999).

In addition to isolated CMEs, further complexity of heliospheric transients comes from the interaction of successive CMEs (Burlaga et al. 2003; Lugaz et al. 2012). In fact, there are a number of fundamental processes which need to be better understood: the deflection of a CME by another, the momentum exchange between colliding CMEs and the fate of shock waves as they propagate inside magnetic ejecta. In this article, we first review observations and modeling of CMEs in the heliosphere, with an emphasis on the need for improved models of TMFRs. Next, we summarize recent progresses in our understanding of CME-CME interaction obtained by combining numerical simulations and the analysis of heliospheric observations and measurements. We conclude with a short discussion of further progresses expected from future missions.

\section{What we really know about CMEs and how we know it}

\subsection{In Situ Measurements}

From the analyses of in situ measurements of CMEs at multiple points came the definition of magnetic clouds, magnetically dominated structures characterized by an enhanced and smoothly varying magnetic field, low proton $\beta$ and temperature as a subset of magnetic ejecta observed at 1 AU (Burlaga et al. 1981). To explain the smooth rotation of the magnetic field, Goldstein (1983) and Marubashi (1986) proposed that magnetic clouds can be described as twisted magnetic flux ropes satisfying the force-free condition. Burlaga (1988) showed that a linear force-free model such as that described by Lundquist (1950) was a good approximation to the in situ data. This resulted in the development of force-free fitting techniques with a twisted flux rope (Lepping et al. 1990), which is still one of the most commonly used techniques to analyze in situ observations of CMEs to date. Improvements upon this technique comprise the inclusion of the expansion of the magnetic cloud (Farrugia et al. 1993; Shimazu \& Vandas 2002) and the possibility of taking into consideration a curved axis (the torus models of Romashets \& Vandas 2003; Marubashi \& Lepping 2007; Janvier et al. 2013; Janvier 2014). Some techniques have also been developed to incorporate the effect of reconnection of the magnetic cloud on its way to Earth (Dasso et al. 2006; Ruffenach et al. 2012).

Other models that dispense with the force-free approximation are the Grad-Shafranov reconstruction ( $\mathrm{Hu} \&$ Sonnerup 2001) based on the magneto-hydrostatic approximation, elliptical non-force-free models (e.g., see Hidalgo et al. 2002) and other non-cylindrical models (Mulligan \& Russell 2001; Owens et al. 2012, among others). Common to all these methods is the assumption of near-invariance along the reconstructed magnetic cloud axis, (2.5-D approximation). Recently, using a numerical simulation of a CME with a field rotation dominated by writhe, Al-Haddad et al. (2011) showed that this approximation, which is central to all frequently used models to reconstruct CMEs, could predict a twisted flux rope even when it is not present. Only spheroidal models (Gosling 1990; Vandas et al. 1993), proposed in the early 1990s, dispense with the assumption; however, they have been all but abandoned by the large majority of researchers. It should be kept 

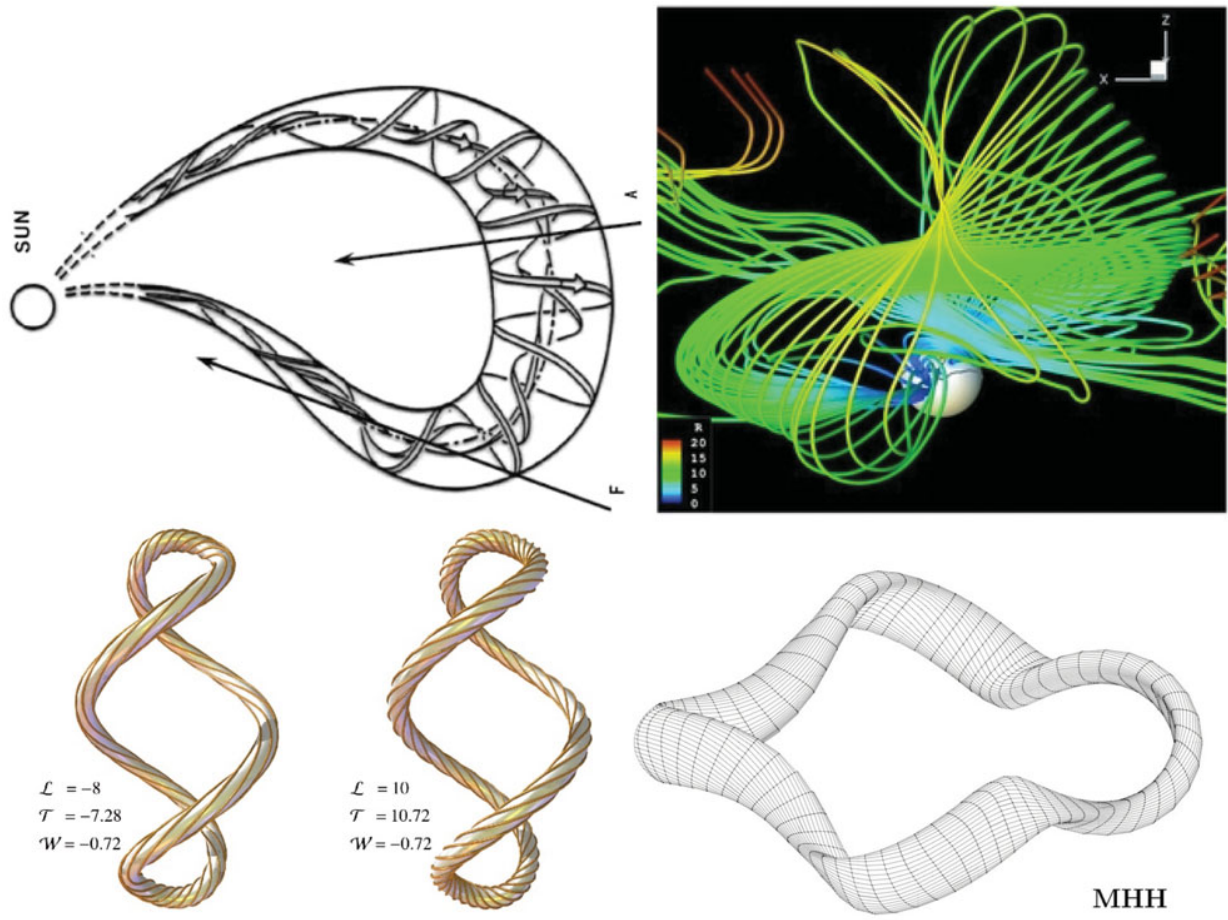

Figure 1. Four different "models" of CMEs. Top left: TMFR (classical picture with a bent axis). Top right: 3-D simulated rotating magnetic field without significant twist. Bottom: Writhed and twisted fields, which cannot yet be analyzed by current reconstruction and fitting models (right from stellarator).

in mind that reconstruction of CMEs from in situ measurements at $1 \mathrm{AU}$ is intrinsically an ill-posed problem (there is no unique solution) even under the assumption that CMEs do not evolve as they pass over the spacecraft. This is because it is necessary to solve a set of $3-\mathrm{D}$ differential equations with only a $1-\mathrm{D}$ boundary condition (obtained from the time series at a single point).

Thanks to the launch of the STEREO spacecraft in 2006, it is, for the first time, possible to routinely and simultaneously probe the same CME at different locations but at almost the same heliocentric distance (within $\pm 10 \%$ of 1 AU). Farrugia et al. (2011) analyzed one such event observed by Wind and the two STEREO spacecraft in November 2007 and found very different orientations of the CME axis depending on which measurements were used. Other multi-spacecraft measurements also resulted in different reconstructed orientations from different spacecraft, but they may be reconciled by considering that one of the two spacecraft measured the "leg" of a flux-rope CME (Möstl et al. 2012; Nieves-Chinchilla et al. 2012), that the global morphology of CMEs is not cylindrically symmetric (Mulligan \& Russell 2001), or by using different techniques (see Démoulin 2014, for another interpretation of the November 2007 CME). Lastly, there has been a number of studies that found that the amount of twist is nearly uniform throughout the CME, in contrast to the Lundquist model (Hu \& Sonnerup 2001; Kahler et al. 2011).

Figure 1 shows the classical picture of a CME used for in situ reconstruction (and also for fitting remote-sensing observations, see next section) as drawn by Marubashi \& Lepping (2007) and inspired by previous works by Burlaga et al., the numerical simulation of Jacobs et al. (2009) without an axial invariance and two examples of twisted and writhed field from Berger \& Prior (2006) and from the stellarator in nuclear fusion (Lyon 
et al. 1997), which might be more appropriate models for CMEs measured in situ. Burlaga et al. (2002), when analyzing a complex ejecta resulting from the merging of multiple CMEs drew an intricate picture of the magnetic field inside CMEs, and drew a parallel to a DNA molecule (their Figures 7 and 8), an example of a structure incorporating twist and writhe.

\subsection{Remote-sensing observations}

Coronagraphic observations of CMEs were first made in the 1970s (MacQueen et al. 1974; Gosling et al. 1975); following comparisons with in-situ measurements, the concept of CMEs as flux ropes was developed and it is currently the accepted paradigm. TMFRs are also a result of flare reconnection during all proposed CME initiation models.

Chen (1996) developed an analytical model of a CME as a flux rope driven by the $\mathbf{j} \times \mathbf{B}$ force. It has been used to understand their evolution in the corona and, recently extended into the heliosphere (Kunkel \& Chen 2010). In addition to this model, visual fitting of CMEs in coronagraph and heliospheric imagers images with a flux rope shape has been performed by a number of researchers (Thernisien et al. 2006; Wood et al. 2009). Other models to analyze remote-sensing observations of CMEs, which do not assume a flux rope shape, include direct triangulation (Liu et al. 2010) and methods based on the idea of a circular or elliptical cross-section of the CME front (Byrne et al. 2009; Lugaz et al. 2009a; Davies et al. 2012). The most advanced of these models fit observations using concave or convex segments of circles (Tappin \& Howard 2009). As for in situ measurements, these reconstructions are not unique, even for a given model. This is because a visual fitting of 6-8 parameters representing the CME is attempted using only 2 or 3 images. For example, CME rotation and over-expansion may be mistaken for one another (as pointed out by Poomvises et al. 2010, for the 2008 March 25 CME). Differences between different analysis techniques can be even more significant.

While not providing heliospheric observations, SDO has been able to reveal the formation of the possible TMFR in the low corona (e.g., Cheng et al. 2011; Su et al. 2012). In SDO images (see for example Zhang et al. 2012), the TMFR may already be strongly distorted by $1.3 R_{\odot}$ to a point which is captured neither by models used to analyze coronagraphic images or in situ measurements, nor by most numerical simulations.

Recent progress has been made thanks to SECCHI observations and, particularly with a new post-processing developed by DeForest et al. (2011). There has been a few observations of CME distortion (Savani et al. 2010), which is expected to be due to the interaction with the bimodal solar wind and has been inferred from in situ measurements (Owens et al. 2006) and predicted from numerical simulations (Riley et al. 2002; Manchester et al. 2004b). CMEs are often observed in heliospheric imagers bounded by two density enhancements, probably associated with their expansion. Taking advantage of these observations, it is possible to determine the radial and temporal evolution of the CME width, its expansion and aspect ratio (Savani et al. 2009; Nieves-Chinchilla et al. 2012; Lugaz et al. 2012). The CME expansion has been found to be proportional to $\mathrm{r}^{0.5-0.9}$, in agreement with statistical studies based on measurements of different CMEs at different distances (Bothmer \& Schwenn 1998; Liu et al. 2005) and also with theoretical studies (Démoulinet al. 2008; Gulisano et al. 2010). In a series of recent works, Howard \& DeForest (2012) and DeForest et al. (2013) have been able to directly link remote-sensing and in situ measurements, to confirm the identification of the magnetic ejecta as the dark cavity in corona graphic and HI images, to determine the CME mass increase during its heliospheric propagation (found to be by a factor of $\sim 2$ as found in numerical simulations Lugaz et al. 2005b) and to identify the different sources of material for the CME sheath region, in part made of coronal material and in part of swept-up 

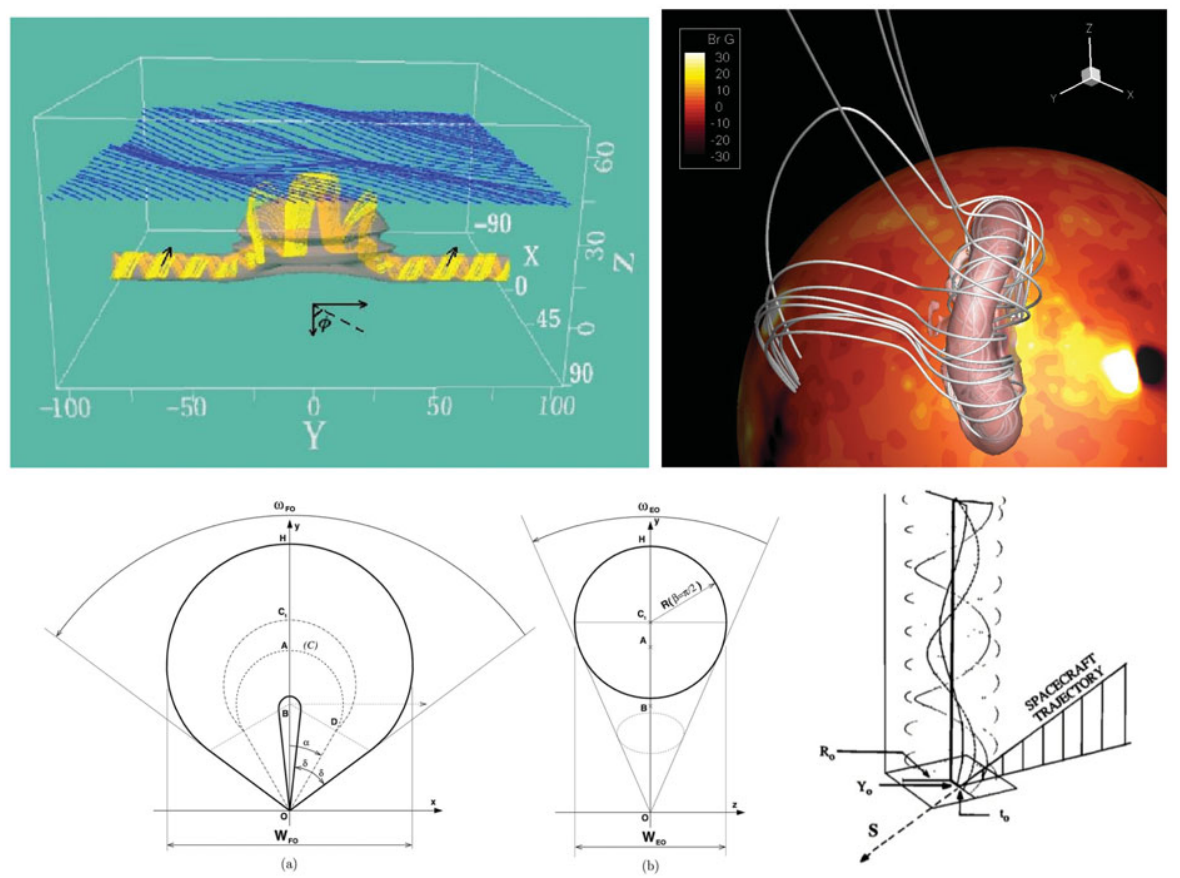

Figure 2. Top: Distorted TMFRs from numerical simulations from Galsgaard et al. (2005) and Lugaz et al. (2011). Bottom: TMFR models used to analyze remote-sensing and in-situ measurements from Thernisien et al. (2006) and Lepping et al. (1990).

solar wind material. Lastly, an area of active research, due in part to its importance for space weather forecasting, is the deceleration of CMEs as they propagate due to their interaction with the solar wind (e.g., see Tappin 2006; Vršnak et al. 2010).

\subsection{Numerical modeling}

Flux ropes initiated at the Sun in numerical models are able to "reproduce" typical in situ measurements of magnetic clouds (Riley et al. 2002; Manchester et al. 2004b; Chané et al. 2006; Shen et al. 2011). These models used an out-of-equilibrium flux rope (Titov \& Démoulin 1999; Roussev et al. 2003) or flux-rope like structures (Gibson \& Low 2000; Manchester et al. 2004b). Numerical simulations have been primarily used to study the initiation of CMEs. In this case, the models include, at least, the study of the loss of equilibrium of the TMFR. Some models start from a TMFR initially in equilibrium and destabilize it with boundary motions, which represent either flux cancellation or flux emergence (Linker \& Mikic 1995; Török et al. 2011), while in other models it emerge from below the photosphere (Archontis et al. 2004; Manchester et al. 2004a; Fan \& Gibson 2004; Roussev et al. 2012). In all these models, the TMFR is initiated with a nondistorted axis, although it may kink or get distorted during the eruption phase (Lugaz et al. 2011; Török et al. 2004) (see top panels of Figure 2). It is one important reason to initiate simulations in the lower corona or even lower down to reproduce as accurately as possible the early phase of eruption and the associated distortion. Other models attempt to create the TMFR from boundary motions (Amari et al. 1996; Lynch et al. 2008; Aulanier et al. 2010; Zuccarello et al. 2012) and study the further destabilization and eruption of the TMFR. In Lugaz \& Roussev (2011), we gave an overview of comparison of numerical simulations with heliospheric images and the interested reader is invited to refer to that review for additional information. 


\subsection{Summary}

There is an obvious dichotomy in our way of understanding CMEs. On the one hand, numerical simulations and the latest observations by SDO, clearly show that TMFRs, when they are present, may be already strongly distorted and kinked in the low corona (see top panels of Figure 2) to a point where it is hard to relate local and global properties such as the orientation of the CME "axis" or the width of the CME. On the other hand, most models to analyze heliospheric observations and in situ measurements of CMEs are based on non-writhed structures with an invariance along the TMFR axis (see bottom panels of Figure 2). Most models do not even allow for deformation of the TMFRs beyond an elliptical cross-section. It should also be noted that properties of TMFRs reconstructed from coronal observations often differ drastically from the properties of flux ropes reconstructed from in situ measurements (Yurchyshyn et al. 2001; Isavnin et al. 2013). A better understanding of CMEs in the heliosphere may require a shift in paradigm by acknowledging the complexity of their evolution and the development of models combining remote-sensing observations and in situ measurements, with input from modeling, in order to reconcile local and global properties of CMEs.

\section{CME-CME Interaction}

Another source of complexity in the heliosphere is the interaction of successive CMEs. With an average of more than three coronal mass ejections (CMEs) per day near solar maximum, CME-CME interaction should occur regularly in the inner heliosphere. Some of the earliest reports of likely CME-CME interaction were associated with the series of events in early August 1972 (Ivanov 1982), as well as multi-sapcecraft measurements of series of CMEs during the Helios era (Burlaga et al. 1987). With the improvement of coronagraph observations, and specifically the large field-of-view of LASCO/C3 covering distances up to $32 R_{\odot}$, the 1990 s witnessed the first direct observations of CME-CME interaction (Gopalswamy et al. 2001) and further confirmations that they are associated with complex ejecta or compound streams at 1 AU (Burlaga et al. 2003; Wang et al. 2002). It has also been proposed that some seemingly isolated CMEs measured in situ may in fact result from the interaction of multiple CMEs on their way to Earth (Dasso et al. 2009). The past six years have seen a similar increase in detection of CME-CME interaction thanks to the wide field-of-view of the HIs onboard STEREO (e.g., see Rouillard 2010). Recent HI observations of CME-CME interaction include the 2008 November CMEs (Shen et al. 2012), the 2010 May CMEs (Lugaz et al. 2012) and the 2010 August CMEs (Harrison et al. 2012; Temmer et al. 2012). Analysis of these events often combine remotesensing observations with in situ measurements and sometimes numerical simulations (Lugaz et al. 2009b; Webb et al. 2009, 2013).

Combining numerical simulations, theoretical works and the analysis of in situ and remote-sensing observations, a number of studies have investigated the complex physical processes occurring during instances of CME-CME interaction. The change in speed during the collisions of two CMEs had been previously investigated via numerical simulations (Lugaz et al. 2005a; Xiong et al. 2006). Recently, it has also been investigated by analyzing SECCHI observations, which revealed unexpected behavior of either deceleration of the overtaking CME below the speed predicted from a perfectly inelastic collision (Temmer et al. 2012) or acceleration of the overtaken CME beyond the speed predicted from a perfectly elastic collision (super-elastic, Lugaz et al. 2009a; Shen et al. 2012). This latter phenomenon was then further identified in another numerical simulation (Shen et al. 2013). Recent observations (Lugaz et al. 2012) have also confirmed the numerical results of the deflection of one CME by another as they collide (Schmidt \& 

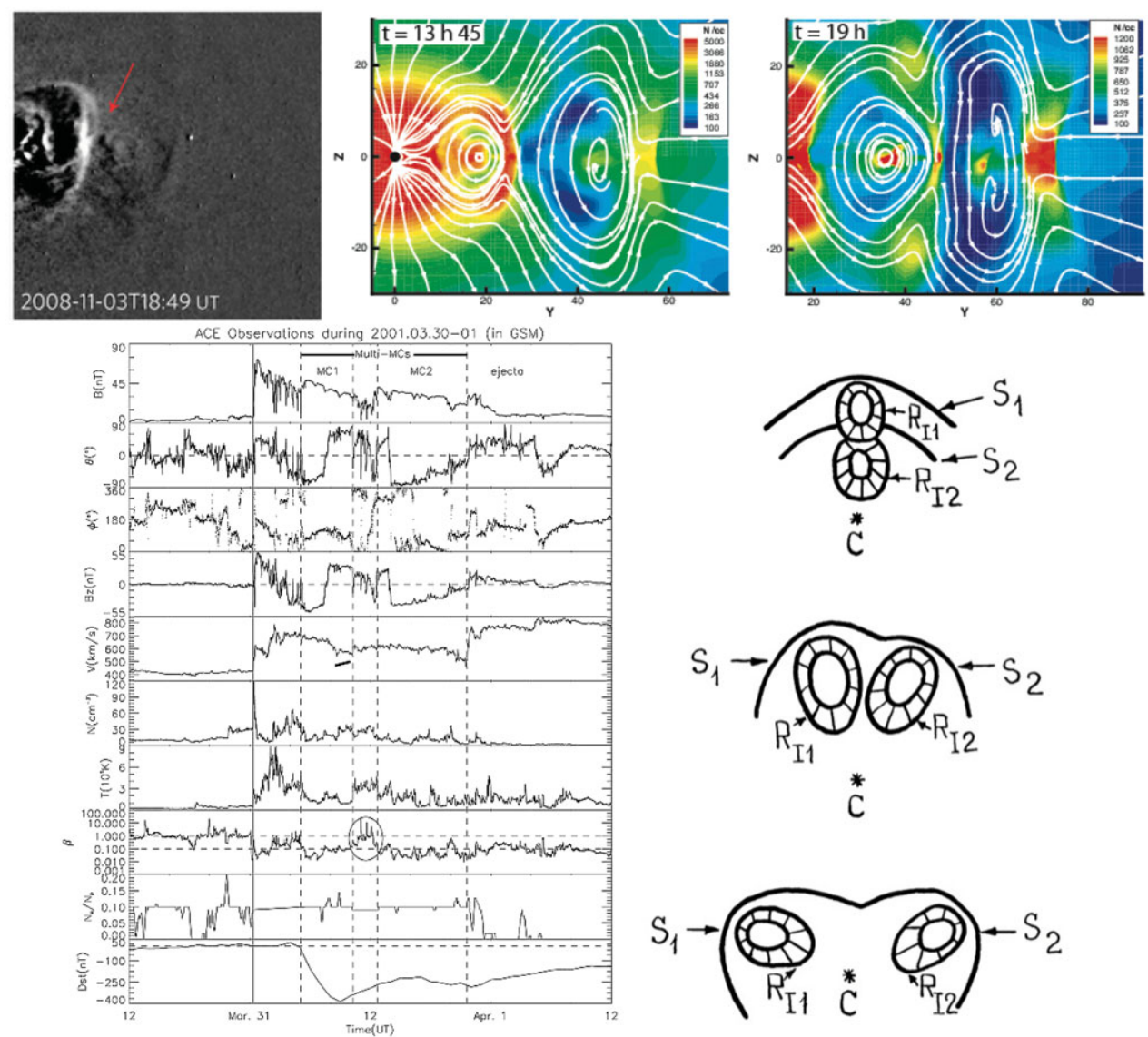

Figure 3. Different manifestations or studies of CME-CME interaction. From top left to bottom right: HI observations (Shen et al. 2012), MHD simulation (Lugaz et al. 2005a), in-situ measurements (Wang et al. 2003) and cartoon representation from the early 1980s (Ivanov 1982).

Cargill 2004; Xiong et al. 2009) and the potential over-expansion of the overtaken CME after its compression (Gulisano et al. 2010, 2012). The potential merging of successive shock waves was already recognized by Ivanov (1982) (see Figure 3) and has been detected from multiple in situ measurements at different heliocentric distances (Farrugia \& Berdichevsky 2004) and studied via numerical simulations (Lugaz et al. 2005a). The effect of successive and interacting CMEs on the acceleration of particles (e.g., see Li et al. 2012) and the potential for sympathetic eruptions (Török et al. 2011; Schrijver \& Title 2011) are also two active areas of research associated with CME-CME interaction but go beyond the scope of this review. Figure 3 illustrates some manifestations and studies of CME-CME interaction.

Overall, a complex but consistent picture of the interaction of two CMEs is starting to emerge. Analyses must move beyond the simple kinematic model to incorporate the CME expansion, the effect of the shock waves as well as the internal magnetic pressure and magnetic tension in the CMEs. Interaction between two CMEs typically takes 6-12 hours and can result in strong compression of the overtaken CME and an increase in its internal magnetic field. If the collision is head-on and the relative orientation of the two CMEs do not allow for full reconnection, this instance of CME-CME interaction is likely to result in a multiple-magnetic cloud event (Wang et al. 2002). However, if the collision further 
results in the deflection of one CME or in a strong reconnection between the two CMEs (akin to full "cannibalism"), the first CME, in a state of enhanced magnetic pressure, is bound to over-expand. We speculate that the enhanced magnetic pressure inside the CME may be converted into kinetic energy through this over-expansion, resulting in a higher speed than expected. Such an event may also be mistaken for an isolated CME at 1 AU (Dasso et al. 2009; Lugaz et al. 2012). Lastly, the interaction of more than two CMEs in a short span can destroy the regularity of the magnetic field in magnetic clouds resulting in complex ejecta (Burlaga et al. 2003). This general picture must be confirmed by performing more dedicated studies of CME-CME interaction as well as series of parametric numerical investigation to evaluate the effect of the CME relative speed, direction and orientation on the interaction process.

\section{Conclusion}

We have reviewed some recent works related to the heliospheric propagation of CMEs, focusing first on what they teach us about the flux rope nature of CMEs. We have emphasized that TMFRs are a sufficient model to understand CMEs, but there is no indication that it is necessary. In addition, and more importantly, most TMFR models used to analyze observations appear overly simplistic as compared to observations, which often reveal bends and writhe as well as a varying cross-section shape along the CME "axis". SECCHI observations are able to reveal the density structure of the magnetic ejecta and the CME sheath, and this global view of the CME should be incorporated into models in order to analyze the local properties of CMEs as provided by in situ measurements. We have also summarized some recent investigations of CME-CME interaction. For the first time, remote-sensing observations can complement in situ measurements and numerical simulations to shed light on the acceleration and deceleration of CMEs following their collision and the changes in the expansion of CMEs. With planned missions such as Solar Probe+ and Solar Orbiter (Müller et al. 2013) providing in situ measurements in the inner heliosphere and upper corona, the next decade should allow us to better study the evolution of CMEs as they propagate, including their interaction, especially if heliospheric remote-sensing imaging is available. Other future missions should include remote-sensing observations from out of the ecliptic plane (as will be provided, partially, by Solar Orbiter). Coronagraphs and heliospheric images from a solar polar orbit may allow us to better understand the longitudinal properties of CMEs: their extent, deflection, the extent of the CME-driven shocks and the shock stand-off distance among others. By combining these observations and measurements and numerical simulations, we should be able to more fully understand the richness and complexity of the heliospheric evolution of CMEs.

\section{Acknowledgements}

We would like to thank S. Poedts, K. Galsgaard and P. Démoulin for useful discussions regarding the nature of CMEs. N. L. would like to thank B. Schmieder and E. Pariat for the great symposium in Paris. The work for this manuscript was supported by the grants AGS1239699, AGS1239704 and NNX13AH94G, as well as the STEREO grant to UNH.

\section{References}

Al-Haddad, N., Roussev, I. I., Möstl, C., et al. 2011, Astrophys. Journ. Lett., 738, L18 Amari, T., Luciani, J. F., Aly, J. J., \& Tagger, M. 1996, Astron. Astrophys., 306, 913 Amari, T., Luciani, J. F., Mikic, Z., \& Linker, J. 1999, Astrophys. Journ. Lett., 518, L57 Antiochos, S. K., DeVore, C. R., \& Klimchuk, J. A. 1999, Astrophys. J., 510, 485 
Archontis, V., et al. 2004, Astron. Astrophys., 426, 1047

Aulanier, G., Török, T., Démoulin, P., \& DeLuca, E. E. 2010, Astrophys. J., 708, 314

Berger, M. A. \& Prior, C. 2006, Journal of Physics A Mathematical General, 39, 8321

Bothmer, V. \& Schwenn, R. 1998, Annales Geophysicae, 16, 1

Burlaga, L. \& Berdichevsky, D., et al. 2003, J. Geophys. Res., 108, 2

Burlaga, L., Sittler, E., Mariani, F., \& Schwenn, R. 1981, J. Geophys. Res., 86, 6673

Burlaga, L. F. 1988, J. Geophys. Res., 93, 7217

Burlaga, L. F., Behannon, K. W., \& Klein, L. W. 1987, J. Geophys. Res., 92, 5725

Burlaga, L. F., \& Plunkett, S. P., St. Cyr, O. C. 2002, J. Geophys. Res., 107, 1

Byrne, J. P. \& Gallagher, P. T., et al. 2009, Astron. Astrophys., 495, 325

Chané, E. \& van der Holst, B., et al. 2006, Astron. Astrophys., 447, 727

Chen, J. 1996, J. Geophys. Res., 101, 27499

Cheng, X., Zhang, J., Liu, Y., \& Ding, M. D. 2011, Astrophys. Journ. Lett., 732, L25

Dasso, S., Mandrini, C. H., Démoulin, P., \& Luoni, M. L. 2006, Astron. Astrophys., 455, 349

Dasso, S., Mandrini, C. H., Schmieder, B., et al. 2009, J. Geophys. Res., 114, 2109

Davies, J. A., Harrison, R. A., Rouillard, A. P., et al. 2009, Geophys. Res. Lett., 36, L02102

Davies, J. A., Harrison, R. A., Perry, C. H., et al. 2012, Astrophys. J., 750, 23

Démoulin, P., Nakwacki, M. S., Dasso, S., \& Mandrini, C. H. 2008, Solar Phys., 250, 347

Démoulin, P. this issue

DeForest, C. E., Howard, T. A., \& McComas, D. J. 2013, Astrophys. J., 769, 43

DeForest, C. E., Howard, T. A., \& Tappin, S. J. 2011, Astrophys. J., 738, 103

Fan, Y. \& Gibson, S. E. 2004, Astrophys. J., 609, 1123

Farrugia, C. \& Berdichevsky, D. 2004, Annales Geophysicae, 22, 3679

Farrugia, C. J., Burlaga, L. F., Osherovich, V. A., et al. 1993, J. Geophys. Res., 98, 7621

Farrugia, C. J., Berdichevsky, D. B., Möstl, C., et al. 2011, J. Atmos. Solar-Terr. Phys., 73, 1254

Galsgaard, K., Moreno-Insertis, F., et al. 2005, Astrophys. Journ. Lett., 618, L153

Gibson, S. E. \& Low, B. C. 2000, J. Geophys. Res., 105, 18187

Goldstein, H. 1983, in NASA Conference Publication, Vol. 228, 731-733

Gopalswamy, N., Yashiro, S., et al. 2001, Astrophys. Journ. Lett., 548, L91

Gosling, J. T. 1990, AGU Geophysical Monograph Series, 58, 343

Gosling, J. T., Hildner, E., MacQueen, R. M., et al. 1975, Solar Phys., 40, 439

Gulisano, A. M., Démoulin, P., Dasso, S., \& Rodriguez, L. 2012, Astron. Astrophys., 543, A107

Gulisano, A. M., Démoulin, P., et al. 2010, Astron. Astrophys., 509, A39

Harrison, R. A., Davies, J. A., Möstl, C., et al. 2012, Astrophys. J., 750, 45

Hidalgo, M. A., Nieves-Chinchilla, T., \& Cid, C. 2002, Geophys. Res. Lett., 29, 130000

Howard, T. A. \& DeForest, C. E. 2012, Astrophys. J., 746, 64

Howard, T. A., Bisi, M. M., Buffington, A., et al. 2013, Space Sci. Rev.

Hu, Q. \& Sonnerup, B. U. Ö. 2001, Geophys. Res. Lett., 28, 467

Isavnin, A., Vourlidas, A., \& Kilpua, E. K. J. 2013, Solar Phys.

Ivanov, K. G. 1982, Space Sci. Rev., 32, 49

Jacobs, C., Roussev, I. I., Lugaz, N., \& Poedts, S. 2009, Astrophys. Journ. Lett., 695, L171

Janvier, M., Démoulin, P., \& Dasso, S. 2013, Astron. Astrophys., 556, A50

Janvier, M. this issue

Kahler, S. W., Krucker, S., \& Szabo, A. 2011, J. Geophys. Res., 116, 1104

Kunkel, V. \& Chen, J. 2010, Astrophys. Journ. Lett., 715, L80

Lepping, R. P., Burlaga, L. F., \& Jones, J. A. 1990, J. Geophys. Res., 95, 11957

Li, G., Moore, R., Mewaldt, R. A., Zhao, L., \& Labrador, A. W. 2012, Space Sci. Rev., 171, 141

Linker, J. A. \& Mikic, Z. 1995, Astrophys. Journ. Lett., 438, L45

Liu, Y., Davies, J. A., Luhmann, J. G., et al. 2010, Astrophys. Journ. Lett., 710, L82

Liu, Y., Richardson, J. D., \& Belcher, J. W. 2005, Planet. Space Sci., 53, 3

Lugaz, N., Downs, C., Shibata, K., et al. 2011, Astrophys. J., 738, 127

Lugaz, N., Farrugia, C. J., Davies, J. A., et al. 2012, Astrophys. J., 759, 68

Lugaz, N., Manchester, W. B., \& Gombosi, T. I. 2005a, Astrophys. J., 634, 651 
—. 2005b, Astrophys. J., 627, 1019

Lugaz, N. \& Roussev, I. I. 2011, J. Atmos. Solar-Terr. Phys., 73, 1187

Lugaz, N., Vourlidas, A., \& Roussev, I. I. 2009a, Annales Geophysicae, 27, 3479

Lugaz, N., Vourlidas, A., Roussev, I. I., \& Morgan, H. 2009b, Solar Phys., 256, 269

Lundquist, S. 1950, Ark. Fys., 2, 361

Lynch, B. J., Antiochos, S. K., et al. 2008, Astrophys. J., 683, 1192

Lyon, J. F., Rome, J. A., et al. 1997, Chapter 2: Final Report of Stellarator Power Plant Study

MacQueen, R. M., Eddy, J. A., Gosling, J. T., et al. 1974, Astrophys. Journ. Lett., 187, L85+

Manchester, W. B., Gombosi, T., DeZeeuw, D., \& Fan, Y. 2004a, Astrophys. J., 610, 588

Manchester, W. B., Gombosi, T. I., Roussev, I., et al. 2004b, J. Geophys. Res., 109, 2107

Marubashi, K. 1986, Adv. Space Res., 6, 335

Marubashi, K. \& Lepping, R. P. 2007, Annales Geophysicae, 25, 2453

Möstl, C., Farrugia, C. J., Kilpua, E. K. J., et al. 2012, Astrophys. J., 758, 10

Müller, D., Marsden, R. G., St. Cyr, O. C., \& Gilbert, H. R. 2013, Solar Phys., 285, 25

Mulligan, T. \& Russell, C. T. 2001, J. Geophys. Res., 106, 10581

Nieves-Chinchilla, T., Colaninno, R., Vourlidas, A., et al. 2012, J. Geophys. Res., 117, 6106

Owens, M. J., Démoulin, P., et al. 2012, Solar Phys., 278, 435

Owens, M. J., Merkin, V. G., \& Riley, P. 2006, J. Geophys. Res., 111, 3104

Poomvises, W., Zhang, J., \& Olmedo, O. 2010, Astrophys. Journ. Lett., 717, L159

Riley, P., Linker, J. A., Mikić, Z., et al. 2002, Astrophys. J., 578, 972

Romashets, E. P. \& Vandas, M. 2003, Geophys. Res. Lett., 30, 200000

Rouillard, A. P. 2010, J. Atmos. Solar-Terr. Phys.

Roussev, I. I., Forbes, T. G., Gombosi, T. I., et al. 2003, Astrophys. Journ. Lett., 588, L45

Roussev, I. I., Galsgaard, K., Downs, C., et al. 2012, Nature Physics, 8, 845

Ruffenach, A., Lavraud, B., Owens, M. J., et al. 2012, J. Geophys. Res., 117, 9101

Savani, N. P., Owens, M. J., et al. 2010, Astrophys. Journ. Lett., 714, L128

Savani, N. P., Rouillard, A. P., Davies, J. A., et al. 2009, Annales Geophysicae, 27, 4349

Schmidt, J. \& Cargill, P. 2004, Annales Geophysicae, 22, 2245

Schrijver, C. J. \& Title, A. M. 2011, J. Geophys. Res., 116, 4108

Shen, C., Wang, Y., Wang, S., et al. 2012, Nature Physics, 8, 923

Shen, F., Feng, X. S., et al. 2011, J. Geophys. Res., 116, A04102

Shen, F., Shen, C., Wang, Y., Feng, X., \& Xiang, C. 2013, Geophys. Res. Lett., 40, 1457

Shimazu, H. \& Vandas, M. 2002, Earth, Planets, and Space, 54, 783

Su, Y., Dennis, B. R., Holman, G. D., et al. 2012, Astrophys. Journ. Lett., 746, L5

Tappin, S. J. 2006, Solar Phys., 233, 233-248

Tappin, S. J. \& Howard, T. A. 2009, Space Sci. Rev., 147, 55

Temmer, M., Vršnak, B., Rollett, T., et al. 2012, Astrophys. J., 749, 57

Thernisien, A. F. R., Howard, R. A., \& Vourlidas, A. 2006, Astrophys. J., 652, 763

Titov, V. S. \& Démoulin, P. 1999, Astron. Astrophys., 351, 707

Török, T., Kliem, B., \& Titov, V. S. 2004, Astron. Astrophys., 413, L27

Török, T., Panasenco, O., Titov, V. S., et al. 2011, Astrophys. Journ. Lett., 739, L63

Vršnak, B., Žic, T., et al. , Astron. Astrophys., 512, A43

Vandas, M., Fischer, S., Pelant, P., \& Geranios, A. 1993, J. Geophys. Res., 98, 11467

Wang, Y. M., Wang, S., \& Ye, P. Z. 2002, Solar Phys., 211, 333

Wang, Y. M., Ye, P. Z., \& Wang, S. 2003, J. Geophys. Res., 108, A10, 1370

Webb, D. F., Howard, T. A., Fry, C. D., et al. 2009, Solar Phys., 256, 239

Webb, D. F., Möstl, C., Jackson, B. V., et al. 2013, Solar Phys., 285, 317

Wood, B. E., Howard, R. A., Plunkett, S. P., \& Socker, D. G. 2009, Astrophys. J., 694, 707

Xiong, M., Zheng, H., \& Wang, S. 2009, J. Geophys. Res., 114, A11101

Xiong, M., Zheng, H., Wang, Y., \& Wang, S. 2006, J. Geophys. Res., 111, A08105

Yurchyshyn, V. B., Wang, H., Goode, P. R., \& Deng, Y. 2001, Astrophys. J., 563, 381

Zhang, J., Cheng, X., \& Ding, M.-D. 2012, Nature Communications, 3

Zuccarello, F. P., Meliani, Z., \& Poedts, S. 2012, Astrophys. J., 758, 117 